\title{
Reumofan Induced Edema
}

\author{
Apurva Akkad ${ }^{\mathrm{a}, \mathrm{b}}$, Justin K. Lui ${ }^{\mathrm{a}}$, Sandra Tirrell ${ }^{\mathrm{a}}$
}

\begin{abstract}
We are presenting a first ever published report in the English literature in a patient presenting with adverse effects of an FDA-banned medicine, Reumofan, which has been marketed to treat rheumatism, joint pain, arthritis and neuralgia. It contains undisclosed traces of dexamethasone, diclofenac and methocarbamol. Some of these adverse effects include hypertension, adrenal insufficiency, gastrointestinal bleeding and sudden death. Our patient presented with progressively worsening edema affecting upper and lower extremities, abdominal wall and face. He was ruled out for venous thrombosis, and his cardiac catheterization and transthoracic echocardiogram exhibited no evidence of heart failure. His edema was successfully treated with diuresis during his hospitalization and on follow-up visit, he was found to be adrenally insufficient and hypotensive on discontinuation of Reumofan. This case illustrates the severe adverse effects that can occur as a result of Reumofan use in a patient using this medicine to treat debilitating joint pain and reinforces the importance of a thorough medication history.
\end{abstract}

Keywords: Reumofan; Edema; Drug toxicity

\section{Introduction}

Use of complementary alternative medicines (CAM) remains common, in the range of $33-66 \%$ of clinic patients [1]. Reports suggest that $28 \%$ of the US population is using complementary medicine to relieve pain [2], which comprises out-of-pocket expenditures of over 34 billion dollars per

\footnotetext{
Manuscript accepted for publication April 14, 2014

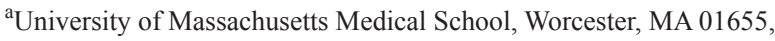
USA

${ }^{\mathrm{b} C}$ Corresponding author: Apurva Akkad, 183 Shrewsbury St Worcester, MA 01604, USA. Email: apurva.akkad@umassmed.edu
}

doi: http://dx.doi.org/10.14740/jem216w year [3]. However, the mechanism of action of many of these medicines is unknown, and the evidence of their efficacy is limited.

\section{Case Report}

A 55-year-old morbidly obese Caucasian gentleman was admitted to the hospital for increasing lower extremity edema. Pertinent past medical history included coronary artery disease, hypertension, obstructive sleep apnea and osteoarthritis. He had been seen in the primary care office 5 days prior to hospital admission and was started on torsemide as well as spironolactone for his edema. The patient noted at least 4 - 5 months of increasing edema, affecting his upper and lower extremities, abdominal wall and face. He had gained 30 pounds in the preceding 3 months without any recent dietary changes. He denies any orthopnea or chest pain, but endorsed some dyspnea on exertion. Home medications included: aspirin, calcium citrate, cyclobenzaprine, fluticasone inhaler, ibuprofen, lisinopril, lovastatin, magnesium, vitamin D, meclizine prn, Percocet prn, spironolactone, torsemide, tamsulosin and amlodipine.

His vital signs were stable with a blood pressure of $144 / 70$, heart rate of 64 and oxygen saturation of $94 \%$ on room air. On physical examination, he was a morbidly obese gentleman with marked edema in his upper and lower extremities as well as his neck and face. Lungs were clear and heart sounds were distant but unremarkable. Abdomen was soft and non-distended. Basic metabolic panel, complete blood count, total protein, albumin, liver function tests, cardiac enzymes and thyroid stimulating hormone were all within normal limits. Brain natriuretic peptide was $20 \mathrm{pg} /$ $\mathrm{mL}$. ECG showed a normal sinus rhythm with a right bundle branch block unchanged from prior as well as no dynamic ST changes. An echocardiogram was poor quality due to body habitus, but had grossly normal ventricular function. Chest X-ray (Fig. 1) and CT chest (Fig. 2) showed no evidence of pulmonary edema, pneumonia, or pulmonary embolism. Venous duplex ruled out acute venous thrombosis.

The patient was started on intravenous furosemide, and on the second day of admission, the patient asked the medi- 


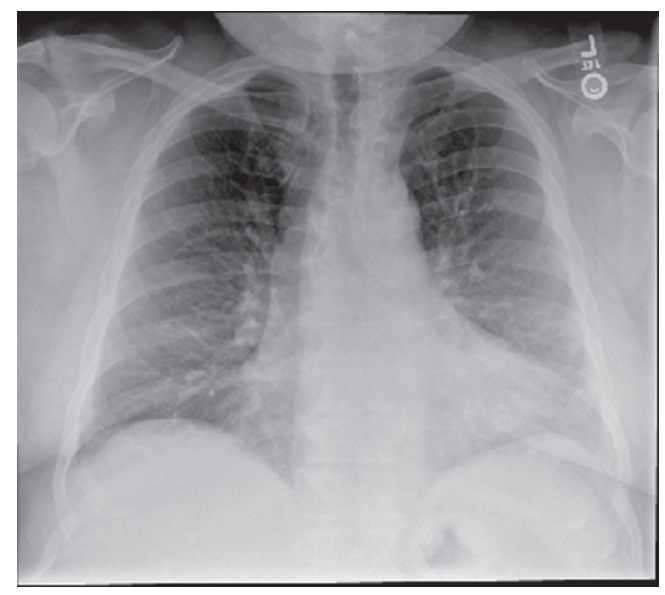

Figure 1. Chest $X$-ray. The lungs are clear without focal consolidation, pleural effusion, or pneumothorax. Normal pulmonary vasculature. Apparent enlargement of the cardiac silhouette is seen to be secondary to prominent pericardiac fat, as identified on the recent CT.

cal team for a list of possible etiologies for his edema. When steroids were mentioned as a potential non-cardiac cause, the patient admitted that he was indeed taking a medicine called Reumofan, which he knew reportedly contained a steroid. He had been using Reumofan for several months now which he stated was available from Mexico via the internet. While on this medication, his chronic pain had resolved and his energy had improved.

After an extensive inpatient and outpatient workup, heart failure and low albumin states were effectively ruled out as the etiology of the patient's edema, which was finally attributed to his use of Reumofan. The patient was diuresed with furosemide and remained in the hospital for a total of 3 days with removal of $2 \mathrm{~L}$ of fluid. He asked to be discharged on his third day of hospitalization despite still having significant peripheral edema. He was subsequently discharged on his home regimen of torsemide, which he felt had been effective in the few days prior to admission. He was instructed to follow-up with his primary care physician for tapering of his Reumofan out of concern for adrenal suppression. On follow-up after his hospitalization, he was found to have a morning cortisol level of $5.5 \mu \mathrm{g} / \mathrm{dL}$ with a systolic blood pressure in the $80 \mathrm{~s}$. He also underwent a coronary angiography which showed no evidence of obstructive coronary artery disease, normal left ventricular end-diastolic pressure and a calculated left ventricular ejection fraction of $75 \%$. Right atrial pressure was in the upper limit of normal. There was no evidence of either diastolic or systolic dysfunction.

\section{Discussion}

Different forms of CAM continue to be an integral compo-

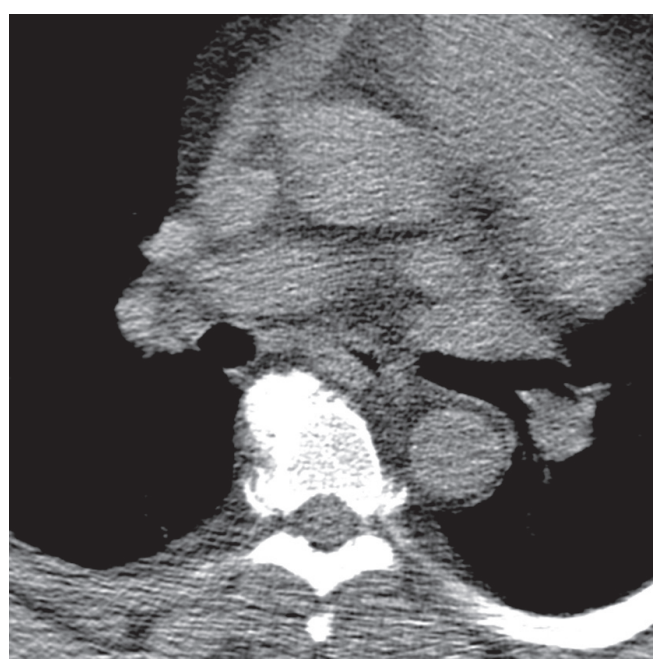

Figure 2. CT chest. There is no large central pulmonary embolism. There is depended bibasilar and subsegmental atelectasis. The lungs are otherwise clear without focal consolidation, pleural effusion, or pneumothorax. There is no hilar lymphadenopathy. There are no mass lesions. The thoracic aorta and its major mediastinal branch vessels are normal throughout their entire course. The mediastinum is unremarkable without lymphadenopathy or pericardial effusion.

nent of outpatient as well as inpatient practice and continue to be beyond the reach of regulation to ensure safety and quality. CAM may be dangerous due to adulteration of the product, as in the case of our patient, interactions with prescribed medications, inherent toxicity, or contamination [2]. In one Singaporean study of 627 cases of adverse effects attributed to CAM products, the most common drugs detected in herbal supplements were sildenafil, dexamethasone, Nnitrosofenfluramine, chlorpheniramine, phenylbutazone, glibenclamide, paracetamol, sibutramine, indomethacin and prednisolone [2]. Despite widespread use, it is estimated that $55-69 \%$ of older patients do not disclose this information to their primary care physician $[4,5]$.

Reumofan has been marketed via the internet as a natural nutritional supplement for the treatment of joint pain and arthritis. Adverse effects include nausea, vomiting, fatigue, hypotension, edema, hypertension, hyperglycemia, adrenal insufficiency, gastrointestinal bleeding and sudden death [6, 7]. The FDA has since recognized the significant adverse effects of Reumofan and has banned its sale in the United States. However, it remains accessible over the internet. Reumofan's adverse effects as well as its pain-relieving effects are attributed to undisclosed active ingredients, diclofenac, dexamethasone and methocarbamol. This report highlights some of the hidden dangers and diagnostic dilemma in the use of readily-available CAM to patients and reinforces the importance of obtaining a thorough medication history including over-the-counter medicines, herbs and nutritional supplements. 


\section{Conflict of Interest}

The authors have no conflicts of interest to disclose.

\section{References}

1. Herman CJ, Allen P, Hunt WC, Prasad A, Brady TJ. Use of complementary therapies among primary care clinic patients with arthritis. Prev Chronic Dis. 2004;1(4):A12.

2. Patel DN, Low WL, Tan LL, Tan MM, Zhang Q, Low MY, Chan CL, et al. Adverse events associated with the use of complementary medicine and health supplements: an analysis of reports in the Singapore Pharmacovigilance database from 1998 to 2009. Clin Toxicol (Phila). 2012;50(6):481-489.

3. Herman PM, Craig BM, Caspi O. Is complementary and alternative medicine (CAM) cost-effective? A systematic review. BMC Complement Altern Med. 2005;5:11.

4. Cheung CK, Wyman JF, Halcon LL. Use of complementary and alternative therapies in community-dwelling older adults. J Altern Complement Med. 2007;13(9):9971006.

5. Cheung C, Geisler C, Sunneberg J. Complementary/alternative medicine use for arthritis by older women of urban-rural settings. J Am Assoc Nurse Pract. 2013.

6. FDA. Reumofan Plus: Recall - Undeclared Drug Ingredient. March 14, 2014. <http://www.fda.gov/Safety/ MedWatch/SafetyInformation/SafetyAlertsforHumanMedicalProducts/ucm306360.htm>. Accessed March 21, 2014.

7. FDA Warning: Dietary Supplement Reumofan Plus May Contain Harmful Drugs. Natural Standard, June 2012. $<$ http://naturalstandard.com/news/news201206005. asp.> Accessed March 21, 2014. 